\title{
Palivizumab e virus respiratorio sinciziale: una panoramica
}

Paolo Manzoni ${ }^{(1)}$

\begin{abstract}
Respiratory Syncytial Virus (RSV) is a very frequent cause of respiratory infections in the first two years of life. Symptoms often are mild to moderate, but in some high-risk categories of infants, particularly prematures and children with bronchopulmonary dysplasia or congenital heart disease, RSV can cause severe lower respiratory tract infections with need of hospitalisation, and, sometimes, even death.

No effective treatment is available, and specific vaccines, despite several attempts during the last decades, do not exist. Palivizumab is a humanised monoclonal antibody targeting specific viral mechanisms controlling infection. If administered intramuscularly monthly during the RSV season as prophylaxis to high risk patients $<2$ years of age, this antibody effectively reduces hospitalisation rates and severity of RSV infection. Based on the data from the two main phase III trials conducted so far, palivizumab prophylaxis results in $45 \%$ to $55 \%$ reduction of hospitalisation rate, with a very satisfactory profile of tolerability as most commonly reported adverse events where transient and mild irritability, diarrhoea or fever.
\end{abstract}

Keywords: Palivizumab; Respiratory Syncytial Virus; RSV prophylaxis

Farmeconomia e percorsi terapeutici 2011; 12(Suppl 3): 3-16

\section{INTRODUZIONE}

Il virus respiratorio sinciziale (RSV) è un virus a RNA molto comune che provoca malattie respiratorie delle alte e basse vie in oltre il $90 \%$ dei bambini sotto i 2 anni di vita. Mentre in soggetti oltre i due anni di età RSV causa in genere lievi sintomi simil-influenzali, in lattanti e neonati prematuri, con basso peso alla nascita e/o con cardiopatia congenita, broncodisplasia polmonare (BPD), fibrosi cistica e immunodeficienza può essere responsabile di malattia respiratoria severa o persino di morte.

Non esistono cure specifiche, al di là del trattamento sintomatico, e sinora i tentativi di produrre un vaccino non sono andati a buon fine. Inoltre gli anticorpi che si generano dopo la prima infezione non sono in grado di evitarne una seconda, ma solo di attenuarne i sintomi, pertanto spesso il bambino va incontro a ricadute di malattia.

In questa cornice si inserisce palivizumab, un anticorpo monoclonale utilizzato come profilassi passiva nelle categorie a rischio, che ha dimostrato efficacia nel ridurre la severità della malattia soprattutto in termini di incidenza e giorni di ospedalizzazione.

\section{L'INFEZIONE DA RSV}

La prima descrizione dell'infezione da RSV risale al 1850 [1], ma solo nel 1955 il virus è stato isolato [2]. Da quel momento, riconosciuta l'importanza della scoperta, sono fioriti i lavori scientifici volti a caratterizzarlo [3].
Oggi si sa che si tratta di un virus a RNA che fa parte della famiglia delle Paramyxoviridae ed è riconosciuto come il più importante agente eziologico delle infezioni respiratorie acute nei bambini con meno di due anni di età [4-6].

La trasmissione del virus si verifica tramite contatto diretto o mediato da superfici contaminate tra le mucose di occhi, bocca o naso di una persona sana e le secrezioni respiratorie di un soggetto infetto [7]. RSV utilizza due glicoproteine (le stesse in grado di generare una risposta anticorpale nell'uomo) presenti sulla superficie dell'envelope (pericapside) per infettare le cellule dell'ospite: la proteina $\mathrm{G}$, responsabile dell'adesione, e la proteina $\mathrm{F}$, che consente la fusione tra l'envelope virale e la membrana plasmatica della cellula da infettare, con conseguente formazione di un sincizio.

Il virus RSV presenta una stagionalità epidemica che varia a seconda delle aree geografiche: nelle zone a clima temperato la stagione di RSV dura dall'inverno alla primavera, in quelle con clima rigido inizia in autunno e termina con l'inverno, mentre nelle zone tropicali coincide con le stagioni delle piogge [8-10].

Si è detto come nei soggetti oltre i due anni di età il virus causi lievi sintomi simil-influenzali, colpendo per lo più le vie aeree superiori; al contrario, una percentuale importante di pazienti con età $<2$ anni presenta invece coinvolgimento delle basse vie aeree [7] che può sfociare, nello specifico nei quadri clinici di cui alla Tabella I.
(I) Divisione di Neonatologia e TIN, Ospedale S. Anna, Torino

Corresponding author

Paolo Manzoni

paolomanzoni@hotmail.com 


\begin{tabular}{ll}
\hline $\begin{array}{l}\text { Bronchite } \\
\text { asmatiforme }\end{array}$ & $\begin{array}{l}\text { Malattia acuta caratterizzata da tosse, ronchi e wheezing } \\
\text { Bronchiolite }\end{array}$ \\
& $\begin{array}{l}\text { Presenza di dispnea sibilante, tachipnea, evidenza } \\
\text { roentgenografica di iperinflazione polmonare con o senza } \\
\text { aree di collasso }\end{array}$ \\
Polmonite & $\begin{array}{l}\text { Rilevamenti radiografici di coinvolgimento del parenchima } \\
\text { polmonare con infiltrati interstizio-alveolari e/o } \\
\text { consolidamento }\end{array}$ \\
\hline
\end{tabular}

\section{Tabella I}

Classificazione delle manifestazioni cliniche di infezione da virus respiratorio sinciziale. Modificato da $[6,11]$

* wheezing $=$ rumore respiratorio di vario tipo (sibilo o ronco) percepito in caso di asma, stenosi bronchiale o presenza di corpi estranei nella trachea o nei bronchi [12]
Lo studio RADAR ha analizzato i dati relativi a tutti $\mathrm{i}$ bambini con età inferiore a 2 anni ospedalizzati in 32 centri italiani a causa di infezioni respiratorie delle vie aeree inferiori dal $1^{\circ}$ novembre 1999 al 30 aprile 2000 . Su un totale di 1.232 soggetti, il $40,6 \%$ di questi risultava positivo a RSV [6]. È anche stato possibile determinare l'andamento epidemico dell'infezione, con un picco a febbraio (prevalenza di infezione da $\mathrm{RSV}=52,2 \%$ dei bambini ospedalizzati), e una prevalenza significativamente inferiore rispetto a tutti gli altri mesi nel mese di novembre (prevalenza di infezione da $\mathrm{RSV}=14,1 \%$ dei bambini ospedalizzati).

Tra i bambini positivi a RSV, il $22,5 \%$ ha avuto una diagnosi di bronchite sibilante, il $48,4 \%$ di bronchiolite e il $26,1 \%$ di polmonite.

La prevalenza di positività a RSV in questa coorte di pazienti ospedalizzati è stata significativamente superiore nei bambini più piccoli, con diminuzioni progressive con l'aumento dell'età:

- 0-3 mesi $=47,4 \%$;

- 4-6 mesi $=41,3 \%$;

- 7-12 mesi = 39,3\%;

- 13-24 mesi $=27,4 \%$.

Questo dato conferma la tendenza al verificarsi di quadri clinici più gravi per l'appunto nei primi mesi di vita.

Inoltre i bambini prematuri sembrano più facilmente colpiti dall'infezione, in quanto, tra i soggetti RSV-positivi, la prevalenza dei nati con età gestazionale $<36$ settimane era del $9,6 \%$ : i dati nazionali più recenti riportano un prevalenza nazionale di nati con età gestazionale compresa tra 33 e 35 settimane del 5\% [25]. Tra i bambini con bronchiolite, la percentuale di pazienti con RSV è risultata essere crescente al decrescere dell'età gestazionale. L'infezione da RSV è stata anche associata a un'alterazione significativamente maggiore della funzionalità respiratoria (maggiore frequenza respiratoria, minor saturazione d'ossigeno, maggior numero di giorni di ossigenoterapia). L'esposizione al fumo di tabacco era significativamente maggiore nei bambini con bronchiolite rispetto a quelli con bronchite sibilante; inoltre l'ordine di genitura era correlato all'infezione: un numero significativamente maggiore di terzogeniti o quartogeniti era affetto da RSV rispetto ai primogeniti. Anche il peso alla nascita risultava essere correlato all'infezione del tratto respiratorio inferiore, in quanto il $14,4 \%$ della popolazione reclutata pesava alla nascita meno di $2.500 \mathrm{~g}, v s$ un dato nazionale pari a 6,0\% [6]. Inoltre il $4 \%$ di tutti i soggetti analizzati dallo studio RADAR era affetto da cardiopatia, a fronte di un dato di prevalenza nazionale molto inferiore (1\%) [25]. 
Successivamente, 187 bambini precedentemente inclusi nello studio RADAR sono stati seguiti con uno studio osservazionale prospettico denominato "FREE", che ha evidenziato un' elevata frequenza di sintomi respiratori a un anno dalla dimissione nei bambini ospedalizzati per infezione al tratto respiratorio nei primi due anni di vita [26].

Un altro progetto di sorveglianza epidemiologica italiana su RSV è stato intrapreso e denominato "Osservatorio VRS". Questo programma di studio si è strutturato come un programma di sorveglianza epidemiologica multicentrico sulle infezioni alle via aeree inferiori durante tre stagioni epidemiche consecutive: ottobre 2000-aprile 2001, ottobre 2001-aprile 2002 e ottobre 2002-aprile 2003 [25,27]. Tra i 29.681 bambini visitati in questo programma di sorveglianza (sono stati presi in considerazione gli accessi al Pronto Soccorso), il 5\% è stato testato per la presenza di RSV. Tra questi, il 22\% è risultato positivo, ma la percentuale aumentava se venivano considerati solo i bambini poi ospedalizzati (Figura 1). I picchi dell'infezione da RSV si sono verificati a febbraio per gli anni I e III e a marzo per l'anno II. Si è evidenziato un alto tasso di ospedalizzazioni tra i soggetti con infezione da RSV (49\% degli affetti), e si è rilevato che la più comune manifestazione clinica dell'infezione del basso tratto respiratorio da RSV era la bronchiolite.

L'età minore di 1 anno è risultata essere il fattore di rischio più importante per lo sviluppo di infezioni delle basse vie respiratorie da

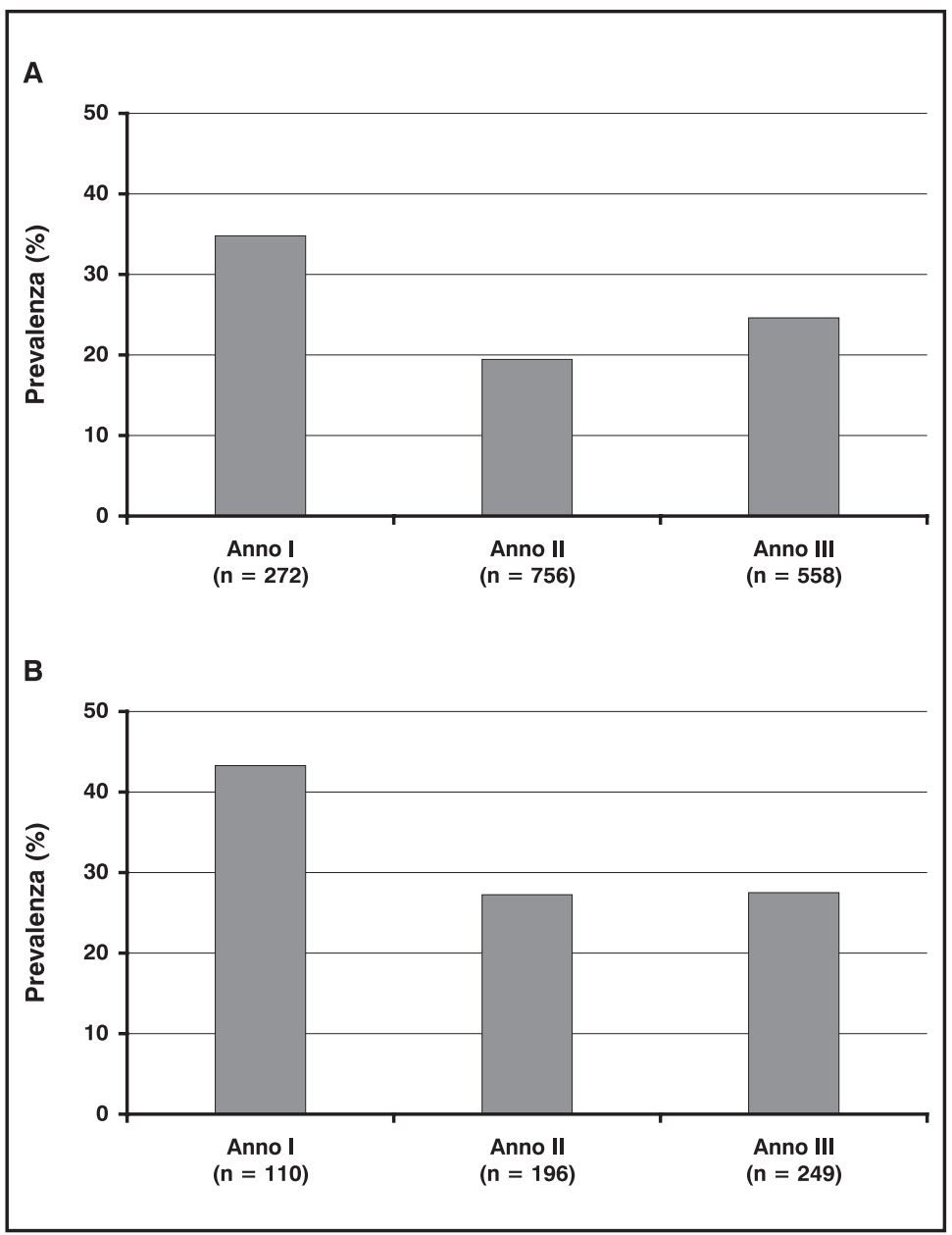

Figura 1

Prevalenza dell'infezione da RSV secondo lo studio Osservatorio VRS nelle 3 stagioni epidemiche considerate. A: prevalenze sul totale dei bambini in studio. B: prevalenze solo sui bambini ospedalizzati (modificato da [25])

\begin{tabular}{|c|c|c|c|}
\hline Fattori di rischio & $\begin{array}{c}\text { Studio RADAR } \\
(n=411) \\
\% \text { soggetti RSV+ }\end{array}$ & $\begin{array}{c}\text { Osservatorio VRS } \\
\text { (ospedalizzati) }(n=172) \\
\% \text { soggetti RSV+ }\end{array}$ & $\begin{array}{c}\text { Osservatorio VRS } \\
\text { (tutti) }(n=355) \\
\% \text { soggetti RSV+ }\end{array}$ \\
\hline Cardiopatia congenita & 11 & 3 & 2 \\
\hline Broncodisplasia & 5 & 8 & 9 \\
\hline \multicolumn{4}{|l|}{ Età gestazionale (settimane) } \\
\hline$\leq 32$ & 5 & 6 & 4 \\
\hline 33-35 & 5 & 4 & 3 \\
\hline \multicolumn{4}{|l|}{ Peso alla nascita (kg) } \\
\hline$<1,5$ & 3 & 4 & 2 \\
\hline $1,5-2,5$ & 12 & 13 & 11 \\
\hline \multicolumn{4}{|l|}{ Età (mesi) } \\
\hline$<3$ & 37 & 33 & 22 \\
\hline $3-6$ & 24 & 23 & 20 \\
\hline $6-12$ & 26 & 24 & 22 \\
\hline Sesso maschile & 61 & 58 & 56 \\
\hline Esposizione al fumo passivo & 35 & 49 & 51 \\
\hline Assenza di allattamento materno & 41 & 32 & 27 \\
\hline Familiarità per atopia & 52 & 45 & 41 \\
\hline Alto ordine di genitura $(\geq 3)$ & 20 & 16 & 17 \\
\hline Elevato numero di fratelli $(\geq 3$ ) & 9 & 7 & 11 \\
\hline Frequenza di comunità scolastiche & 6 & 10 & 16 \\
\hline
\end{tabular}

Tabella II

Fattori di rischio associati a malattia da RSV negli studi RADAR [6] e Osservatorio VRS [27]. La lettera " $n$ " indica il numero di soggetti RSV-positivi (modificato da [25]) 
RSV, da sola o in associazione ad alcuni altri cofattori, quali:

- sesso (maschile);

- bassa età gestazionale (=35 settimane);

- basso peso alla nascita ( $\mathrm{sia}<2,5 \mathrm{~kg}$, sia $<$ $1,5 \mathrm{~kg}$ );

- alto ordine di genitura;

- presenza di fratelli maggiori in famiglia;

- concomitanza di broncodisplasia (BPD) o di malattia polmonare cronica;

- presenza di genitori con allergie;

- particolari condizioni di disagio socioeconomico.

Nella Tabella II sono indicati tutti i fattori di rischio associati a malattia da RSV individuati dal programma di sorveglianza epidemiologica Osservatorio VRS e dallo studio RADAR.

In anni più recenti, un nuovo studio epidemiologico condotto da Di Carlo e colleghi si è posto l'obiettivo di analizzare i dati relativi a 335 dei 705 bambini al di sotto di due anni di età ospedalizzati per infezione delle vie aeree inferiori nell'ospedale infantile "G. Di Cristina" di Palermo tra novembre 2005 e maggio 2006 [28]. Il 53\% di questi è risultato positivo a RSV (178/335). Occorre evidenziare che, come precedente anticipato, la stagione epidemica di RSV varia a seconda delle aree geografica: in effetti a Palermo il picco di infezioni del tratto respiratorio inferiore è stato registrato a marzo, mentre il picco dell'infezione da RSV è stato raggiunto ad aprile, con ben $1^{\prime} 80 \%$ di positività tra la coorte di pazienti considerata. È rilevante notare che, in questo studio, sino a tutto il mese di dicembre non è stata registrato alcun paziente con infezione da RSV. Il 6\% dei pazienti considerati aveva età gestazionale $<36$ settimane (come già ricordato, il dato nazionale attesta il $4 \%$ ). Il 55\% dei bambini con bronchiolite, il $16 \%$ di quelli con polmonite e il $29 \%$ degli affetti da bronchite sibilante risultavano positivi a RSV. Nella Tabella III si riportano le variabili e caratteristiche demografiche che risultavano essere significativamente differenti $(p<0,05)$ tra $i$ bambini positivi a RSV e quelli negativi.

\begin{tabular}{lcc}
\hline & $\begin{array}{c}\text { Positivi a RSV } \\
(\mathbf{n}=\mathbf{1 7 8})\end{array}$ & $\begin{array}{c}\text { Negativi a RSV } \\
(\mathbf{n}=\mathbf{1 5 7})\end{array}$ \\
\hline $\begin{array}{l}\text { Età (mesi) } \\
\text { \% età al momento dell'ospedalizzazione } \\
<6 \text { mesi }(\mathrm{n}=220)\end{array}$ & $3 \pm 1,4$ & $5 \pm 7,1$ \\
Allattamento al seno $(\mathrm{n}=187)$ & 58 & 41 \\
Giorni di ospedalizzazione & $6,4 \pm 3,5$ & 129 \\
Giorni di ossigenoterapia $(\mathrm{n}=235)$ & $4,9 \pm 3,3$ & $4,1 \pm 2,3$ \\
\hline
\end{tabular}

Tabella III

Dati clinici e demografici dei 335 bambini dello studio di Di Carlo (modificato da [28])
L'infezione da RSV risulta associata a un'età inferiore, coerentemente con precedenti segnalazioni che individuano nell'età $\leq 3$ mesi all'inizio della stagione epidemica un forte fattore di rischio per l'infezione severa delle vie aeree inferiori indotta da RSV [25,29,30]. Questo dipende dalle piccole dimensioni delle vie aeree e all'incompleto sviluppo della struttura polmonare, nonché dalla minor probabilità di aver ricevuto dalla madre anticorpi anti-RSV rispetto ai bambini che nascono dopo il picco epidemico di RSV [31]. Questo è anche il motivo per il quale al momento dell'ospedalizzazione una maggior percentuale di bambini RSV-positivi rispetto a quelli RSV-negativi ha meno di 6 mesi di età.

Questi dati confortano anche l'ipotesi che l'allattamento al seno sia un fattore protettivo nei confronti dell'infezione da RSV. Inoltre si evidenzia l'associazione statistica tra infezione da RSV e giorni di ospedalizzazione e di ossigenoterapia, a testimonianza della maggior severità di malattia dei soggetti RSV-positivi.

\section{Lo stato dell'arte sulle opzioni terapeutiche}

Il trattamento per le forme severe da RSV consiste in terapia sintomatica di supporto, che si traduce, sostanzialmente, nel fornire ossigeno al paziente [32]. Sono state studiate numerose altre opzioni terapeutiche, ma sempre con scarso successo. Si è comunque visto che la somministrazione di broncodilatatori può fornire modesti miglioramenti di breve durata in soggetti con bronchiolite da lieve a moderatamente severa [33].

L'unica terapia approvata dall'FDA per la cura della malattia severa da RSV è ribavirina, che pare ridurre la durata della ventilazione meccanica e i giorni di ospedalizzazione [34]. Tuttavia il suo utilizzo è controverso, poiché in alcuni trial non è stata dimostrata la sua efficacia [35-37].

Non esiste, al giorno d'oggi, un vaccino che sia in grado di stimolare il sistema immunitario a reagire contro RSV; persino coloro che hanno già contratto l'infezione sono suscettibili alla reinfezione, anche se di solito essa si manifesta in maniera più lieve $[38,39]$. Per questo motivo alcuni Autori hanno suggerito che l'immunoprofilassi possa soltanto diminuire la severità dell'infezione, non prevenirla [40].

Il primo tentativo di utilizzare un vaccino anti-RSV risale al 1966, pochi anni dopo l'isolamento e la caratterizzazione del virus stesso: si trattava di un virus inattivato con formalina, il cui sviluppo era stato caldeggiato e sponsorizzato dal National Institute for Allergy and Infectious Diseases, che nel 1961 aveva dichiarato che la ricerca sulla malattia da RSV era una priorità. Purtroppo i trial clinici dimostrarono che il vaccino non solo non era 


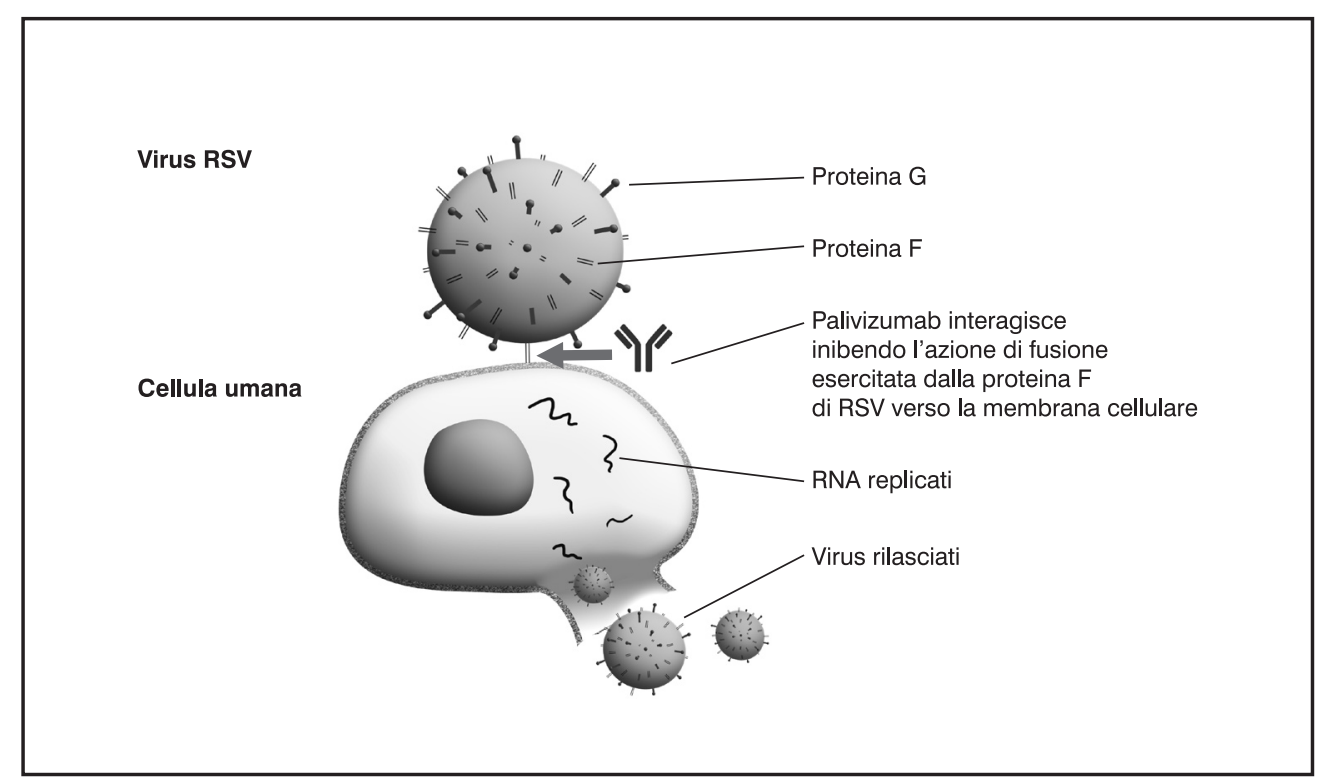

Figura 2

Meccanismo di infezione di $R S V$, e relativa azione di inibizione della fusione esercitata da palivizumab: per infettare le cellule umane RSV utilizza la proteina $G$ presente sull'envelope per aderire alla membrana cellulare e la proteina F per fondersi con essa. Palivizumab inibisce l'azione della proteina $F$, impedendo la fusione e le conseguenti penetrazione del virus, replicazione dell'RNA virale, packaging e rilascio di nuovi virus (che proseguirebbero nel loro processo di infezione di altre cellule) efficace, ma aumentava la severità e il tasso di ospedalizzazioni per infezione da RSV [41-44]. Solo nel 1996 venne immesso in commercio RSV-IGIV, un preparato costituito da anticorpi policlonali anti-RSV indicato come profilassi endovenosa dell'infezione severa delle vie aeree inferiori da RSV in bambini con meno di due anni di età e con broncodisplasia polmonare o storia di prematurità. Benché efficace, come dimostrato dal trial PREVENT [45], presentava alcuni inconvenienti, legati alla durata e al volume dell'infusione (diverse ore e 15 $\mathrm{ml} / \mathrm{kg}$, rispettivamente) e al rischio infettivo legato al fatto che si trattava di un emoderivato. Sulla scorta di risultati ottimi in termini di prevenzione dell'ospedalizzazione da RSV in neonati e bambini ad alto rischio [46], nel 1998 l'anticorpo monoclonale palivizumab venne approvato dall'FDA con la stessa indicazione di RSV-IGIV, e cioè per bambini con Chronic Lung Disease of prematurity o neonati pretermine nati prima della $35^{\circ}$ settimana di gestazione. L'uso approvato era per via intramuscolare. Nel 2003 l'indicazione venne estesa a bambini con cardiopatia congenita emodinamicamente significativa $[3,47]$.

\section{PALIVIZUMAB}

\section{Meccanismo d'azione}

Palivizumab è un anticorpo monoclonale, $\mathrm{e}$ la preparazione commerciale contiene pertanto immunoglobuline identiche tra loro perché prodotte dalla stessa linea cellulare umanizzata (in questo caso la linea NS0 del mieloma murino, che è stata estensivamente caratterizzata).

Si tratta di un anticorpo murino umanizzato, cioè un anticorpo costituito da:

- le Complementary Determining Regions (CDR) di un anticorpo monoclonale murino (che costituiscono circa il 5\% del totale dell'immunoglobulina). Si tratta della frazione di anticorpo deputata al riconoscimento dell'antigene;

- le constant region, che costituisce sostanzialmente 1'“'ossatura dell'anticorpo" e dunque il restante $95 \%$ dell'immunoglobulina, di un IgG1 umana.

L'anticorpo monoclonale palivizumab riconosce la proteina F del virus RSV, esercitando un'azione inibitoria nei confronti della fusione con la cellula da infettare e quindi della conseguente penetrazione del virus (Figura 2). Dato l'alto grado di omologia tra la proteina $\mathrm{F}$ del gruppo A e quella del gruppo B del virus RSV, palivizumab è in grado di svolgere la sua azione contro entrambi i ceppi virali, legandosi alla proteina target con alta affinità $\left(\mathrm{K}_{\mathrm{d}}=0,96 \mathrm{nM}\right)$ [48]. Nel processo di purificazione dell'anticorpo sono osservati e praticati diversi step di rimozione/inattivazione virale.

Palivizumab viene fornito in commercio in forma di prodotto liofilizzato da ricostituire con acqua per preparazioni iniettabili, al fine di ottenere una soluzione $100 \mathrm{mg} / \mathrm{ml}$ da somministrare per via intramuscolare, preferibilmente nella parte anterolaterale della coscia [48]. Il dosaggio raccomandato è di $15 \mathrm{mg} / \mathrm{kg}$ di peso corporeo da praticarsi mensilmente durante la stagione epidemica.

\section{Le indicazioni per l'uso di palivizumab}

Palivizumab è indicato nella prevenzione di gravi affezioni del tratto respiratorio inferiore, che richiedono ospedalizzazione, provocate da RSV in bambini ad alto rischio di malattia da questo virus:

- bambini nati con età gestazionale uguale o inferiore a 35 settimane e con un'età inferiore a 6 mesi al momento dell'inizio del periodo epidemico di RSV; 
- bambini di età inferiore a 2 anni che sono stati trattati per displasia broncopolmonare negli ultimi 6 mesi;

- bambini di età inferiore a 2 anni con malattia cardiaca congenita emodinamicamente significativa [49].

Le più recenti linee guida dell'American Academy of Pediatrics hanno stabilito che $\mathrm{i}$ pazienti eleggibili per la profilassi con palivizumab sono [7]:

- bambini con CLD (Chronic Lung Disease of prematurity) con meno di 24 mesi di età sottoposti a terapia medica per CLD (supplementazione di ossigeno, broncodilatatori, diuretici o terapia cronica con corticosteroidi) entro 6 mesi dall'inizio della stagione epidemica di RSV;

- bambini prematuri nati prima della $32^{\circ}$ settimana di gestazione. Se nati prima della $28^{\circ}$ settimana possono beneficiare della profilassi durante la stagione epidemica entro 12 mesi di vita, se nati tra la $29^{\circ}$ e la $32^{\circ}$ settimana ne possono usufruire entro 6 mesi di vita e, in entrambi i casi, devono continuare la profilassi fino alla fine della stagione;

- bambini prematuri nati tra la $32^{\circ}$ e la $35^{\circ}$ settimana di gestazione, la cui nascita sia avvenuta meno di tre mesi prima della stagione epidemica oppure durante la stagione epidemica, e che abbiano almeno un fattore di rischio aggiuntivo (uno tra la frequentazione di asili nido e la presenza in casa di altri bambini); dopo il compimento di 3 mesi di età la profilassi non è più raccomandata per questa categoria di pazienti; inoltre solo per questa categoria è previsto un massimo di 3 dosi di palivizumab, in luogo delle 5 previste per tutte le altre categorie;
- bambini con anomalie congenite delle vie aeree o malattie neuromuscolari nel primo anno di vita;

- bambini con CHD (Congenital Heart Disease) con meno di due anni di età che ricevono una terapia per controllare l'insufficienza cardiaca congestizia o con ipertensione polmonare da moderata a severa o con cardiopatia cianogena.

Per quanto riguarda la realtà italiana, la SIN - Società Italiana di Neonatologia aveva redatto le prime linee guida nel 2000. A seguito dei risultati di nuovi studi, tali raccomandazioni sono state aggiornate nel 2004 con una pubblicazione su Acta Neonatologica \& Pediatrica e risultano attualmente in vigore [25]. Esse prevedono la somministrazione a cadenza mensile della profilassi con palivizumab alla dose di $15 \mathrm{mg} / \mathrm{kg}$ di peso corporeo a partire da un mese prima dell'inizio della stagione epidemica e durante tutta la stagione invernale (ogni regione, però, deve valutare con cura i dati locali relativi a inizio e fine della stagione epidemica, date le notevoli differenze tra nord e sud Italia, probabilmente dovute a motivi climatici) per un massimo di 5-6 dosi secondo le modalità indicate nella Tabella IV.

\section{Studi di fase I e II su palivizumb}

Tra gli studi di fase I/II condotti su bambini per testare palivizumab in profilassi, citiamo quello di Subramanian e colleghi [50] e quello di Sáez-Llorens e colleghi [51].

Nel primo studio sono stati arruolati soggetti appartenenti a due categorie a rischio:

- bambini prematuri, cioè con $\leq 35$ settimane di età gestazionale e con $\leq 6$ mesi di età;

- bambini con displasia broncopolmonare con $\leq 24$ mesi di età.

\begin{tabular}{|c|c|c|c|}
\hline Indicazione & $\begin{array}{c}\text { Durata della } \\
\text { profilassi }\end{array}$ & $\begin{array}{l}\text { Livello di } \\
\text { evidenza* }\end{array}$ & $\begin{array}{l}\text { Grado di } \\
\text { raccomandazione }\end{array}$ \\
\hline $\begin{array}{l}\text { Età gestazionale } \leq 32 \text { settimane ed età }<1 \text { anno all'inizio della stagione } \\
\text { epidemica }\end{array}$ & 12 mesi & I & $\begin{array}{l}\text { Altamente } \\
\text { raccomandato }\end{array}$ \\
\hline $\begin{array}{l}\text { Bambini con broncodisplasia o malattia polmonare cronica ed età }<2 \text { anni } \\
\text { all'inizio della stagione epidemica }\end{array}$ & 24 mesi & 1 & $\begin{array}{l}\text { Altamente } \\
\text { raccomandato }\end{array}$ \\
\hline $\begin{array}{l}\text { Bambini con cardiopatia congenita emodinamicamente significativa ed età }<2 \\
\text { anni all'inizio della stagione epidemica }\end{array}$ & 24 mesi & 1 & $\begin{array}{l}\text { Altamente } \\
\text { raccomandato }\end{array}$ \\
\hline $\begin{array}{l}\text { Età gestazionale } 33-35 \text { settimane ed età }<1 \text { anno all'inizio della stagione } \\
\text { epidemica, in presenza di altri } 2 \text { fattori di rischio }\end{array}$ & 12 mesi & III & $\begin{array}{l}\text { In presenza di due } \\
\text { fattori di rischio** }\end{array}$ \\
\hline
\end{tabular}

\section{Tabella IV}

Raccomandazioni della Società Italiana di Neonatologia per la profilassi con palivizumab (modificato da [25])

* I = evidenza da studi controllati randomizzati; $\|$-1 = evidenza da studi controllati non randomizzati; II-2 = evidenza da studi analitici di coorte o caso-controllo, preferibilmente effettuati da più di un centro o da più gruppi di ricerca; II-3 = evidenza da confronti tra tempi e luoghi con o senza intervento (risultati eclatanti da studi non controllati possono essere inclusi in questa categoria); III = opinioni di autorità basate sull'esperienza clinica; studi descrittivi o rapporti di gruppi esperti

** Fattori di rischio: dimissione dall'ospedale dopo la nascita durante il periodo epidemico; basso peso alla nascita $\left(<2,5 \mathrm{~kg} 0<10^{\circ}\right.$ percentile); esposizione a fumo passivo; assenza di allattamento al seno; familiarità per atopia; esposizione a fonti di elevato inquinamento atmosferico; nascita da gravidanza multipla (parto gemellare); presenza di fratelli più grandi in famiglia; frequenza di comunità scolastiche; patologie concomitanti gravi (fibrosi cistica, malformazioni della gabbia toracica, malattie neuromuscolari, immunodeficienze, malattie ematologiche, neoplasie); residenza in località remota dove l'accessibilità alle strutture sanitarie è difficoltosa 
Il trial, condotto in più centri e in doppio cieco, ha randomizzato 42 partecipanti a ricevere palivizumab da somministrare per via endovenosa e 20 a ricevere placebo. Il gruppo palivizumab è stato ulteriormente suddiviso in 3 sottogruppi, che ricevevano rispettivamente un dosaggio di 3, 10 o $15 \mathrm{mg} / \mathrm{kg}$ ogni 30 giorni per un massimo di 5 dosi.

L'emivita del preparato è risultata comparabile a quella di altre preparazioni con IgG. La profilassi con palivizumab è risultata sicura e ben tollerata in questa popolazione e non ha indotto una risposta specifica anti-palivizumab.

Negli studi preclinici sui cotton rats si era osservato che la concentrazione ematica che permetteva di ottenere il $99 \%$ di riduzione dei livelli di RSV polmonare era $\geq 40 \mu \mathrm{g} / \mathrm{ml}$.

In questo studio di Subramanian e colleghi tale concentrazione sierica media è stata mantenuta nei soggetti che avevano ricevuto $10 \mathrm{e}$ $15 \mathrm{mg} / \mathrm{kg} / \mathrm{mese}$; nella maggior parte di coloro che avevano ricevuto $15 / \mathrm{mg} / \mathrm{mese}$ di palivizumab la concentrazione è stata mantenuta $\geq 40$ $\mu \mathrm{g} / \mathrm{ml}$ [50]. A 30 giorni dalla prima infusione il sottogruppo $15 \mathrm{mg} / \mathrm{kg}$ aveva concentrazioni sieriche minime medie di $60,6 \mu \mathrm{g} / \mathrm{ml}$, dopo la seconda di $70,7 \mu \mathrm{g} / \mathrm{ml}$, tra 88 e $96 \mu \mathrm{g} / \mathrm{ml}$ nelle successive.

Lo studio di Sáez-Llorens e colleghi ha invece testato la formulazione intramuscolare di palivizumab in un open-label setting costituito da:

- 41 bambini con $\leq 35$ settimane di età gestazionale e con $\leq 6$ mesi di età;
- 24 bambini con displasia broncopolmonare con $\leq 24$ mesi di età.

Nello studio, anch'esso condotto in più centri, sono stati somministrati mensilmente da 1 a 5 tra i seguenti dosaggi di palivizumab: $5 \mathrm{mg} / \mathrm{kg}$ $(\mathrm{n}=11), 10 \mathrm{mg} / \mathrm{kg}(\mathrm{n}=6)$ e $15 \mathrm{mg} / \mathrm{kg}(\mathrm{n}=48)$. La farmacocinetica di palivizumab è risultata essere simile a quella di altre preparazioni immunoglobuliniche. Le concentrazioni sieriche medie di palivizumab sono state di $91,1 \mu \mathrm{g} / \mathrm{ml}$ (range $=52,3-174,0)$ due giorni dopo la dose iniziale di $15 \mathrm{mg} / \mathrm{kg}$ e di $49,2 \mu \mathrm{g} / \mathrm{ml}$ (range = $13,5-132,0)$ a 30 giorni. Sempre all'interno del sottogruppo $15 \mathrm{mg} / \mathrm{kg}$ le concentrazioni sieriche minime medie sono risultate, similmente alla formulazione $\mathrm{EV}$, pari a $70 \mu \mathrm{g} / \mathrm{ml}$. La profilassi è risultata sicura e ben tollerata: solo tre bambini hanno avuto eventi avversi giudicati correlabili a palivizumab. Dieci bambini hanno avuto riscontro di transitori e bassi titoli anticorpali anti-palivizumab non correlati a eventi avversi specifici né ad alterazioni della concentrazione di palivizumab. Due soggetti che avevano ricevuto la dose più bassa di profilassi sono stati ospedalizzati per infezione da RSV, mentre nessuno dei bambini che avevano ricevuto le due dosi più alte è stato ospedalizzato.

In conclusione la dose $15 \mathrm{mg} / \mathrm{kg}$ di palivizumab IM ha mantenuto concentrazioni sieriche minime medie $>40 \mu \mathrm{g} / \mathrm{ml}$.

\section{L'efficacia}

Lo studio di fase III che ha determinato l'immissione in commercio di palivizumab è stato

\begin{tabular}{|c|c|c|c|c|}
\hline & Gruppo placebo & Gruppo palivizumab & Riduzione (\%) & $\mathrm{p}$ value \\
\hline Ospedalizzazione da RSV (incidenza) & $53 / 500(10,6 \%)$ & $48 / 1.002(4,8 \%)$ & 55 & $<0,001$ \\
\hline Giorni totali di ospedalizzazione per RSV & $62,6 / 100$ bambini & $36,4 / 100$ bambini & 42 & $<0,001$ \\
\hline Giorni con aumentato ossigeno supplementare & $50,6 / 100$ bambini & 30,3/100 bambini & 40 & $<0,001$ \\
\hline Giorni con LRI score* $\geq 3$ & 47,4/100 bambini & 29,6/100 bambini & 38 & $<0,001$ \\
\hline Ospedalizzazioni in unità di terapia intensiva (\%) & 3 & 1,3 & 57 & $=0,026$ \\
\hline Giorni totali di permanenza nell'unità di terapia intensiva & 12,7/100 bambini & 13,3/100 bambini & - & $=0,023$ \\
\hline Incidenza di ventilazione meccanica (\%) & 0,2 & 0,7 & - & $=0,280$ \\
\hline Giorni totali di ventilazione meccanica & 1,7 & 8,4 & - & $=0,210$ \\
\hline Incidenza di qualsiasi tipo di ospedalizzazione (\%) & 31 & 24 & 23 & $=0,011$ \\
\hline Giorni totali di qualsiasi tipo di ospedalizzazione & 242/100 bambini & 191/100 bambini & 21 & $=0,005$ \\
\hline Incidenza di ospedalizzazione per motivi respiratori (\%) & 22 & 16 & 27 & $=0,008$ \\
\hline Giorni totali di ospedalizzazione per motivi respiratori & 180/100 bambini & 124/100 bambini & 31 & $=0,004$ \\
\hline $\begin{array}{l}\text { Incidenza di ospedalizzazione per motivi respiratori non } \\
\text { correlati a RSV (\%) }\end{array}$ & 14 & 13 & - & $=0,470$ \\
\hline $\begin{array}{l}\text { Giorni totali di ospedalizzazione per motivi respiratori non } \\
\text { correlati a RSV }\end{array}$ & 118/100 bambini & $88 / 100$ bambini & - & $=0,369$ \\
\hline Otite media (\%) & 40 & 42 & - & $=0,505$ \\
\hline
\end{tabular}

\section{Tabella V}

Riassunto dei principali risultati dello studio IMpact-RSV [46]

* II Lower Respiratory tract IIIness/Infection Score viene utilizzato per valutare la severità della malattia respiratoria; può assumere i seguenti valori: 0 = assenza di malattia/infezione respiratoria; 1 = malattia/infezione delle vie aeree superiori; $2=\mathrm{LRI}$ lieve; $3=\mathrm{LRI}$ moderata; $4=\mathrm{LRI}$ severa; 5 = ventilazione meccanica 
lo studio IMpact-RSV [46]. Si è trattato di uno studio in doppio cieco condotto in 139 centri di USA, Gran Bretagna e Canada che ha randomizzato 1.502 bambini a ricevere o 5 iniezioni IM di palivizumab $15 \mathrm{mg} / \mathrm{kg}$ o placebo (2:1). I bambini venivano reclutati se presentavano le seguenti caratteristiche:

- età gestazionale $\leq 35$ settimane e età $\leq 6$ mesi;

- età $\leq 24$ mesi con broncodisplasia polmonare che richiede un trattamento medico (es. ossigeno supplementare, steroidi, broncodilatatori o diuretici negli ultimi 6 mesi).

L'iniezione veniva effettuata ogni 30 giorni e i bambini venivano seguiti per 150 giorni, cioè fino a 30 giorni dall'ultima iniezione. L'endpoint primario era il ricovero ospedaliero per infezione da RSV (ospedalizzazione da RSV); tra gli ospedalizzati di entrambi i gruppi sono poi stati valutati altri parametri, indicativi di severità dell'infezione. I risultati di tali analisi sono riassunti nella Tabella V.

La profilassi con palivizumab è stata associata a una riduzione del $55 \%$ di ospedalizzazioni da RSV $(p=0,00004)$. Tale riduzione è risultata significativa anche nei sottogruppi dei pazienti affetti da broncodisplasia polmonare (riduzione del $39 \%, \mathrm{p}=0,038$ ) e nei prematuri (riduzione del 78\%, $\mathrm{p}<0,001$ ), così come tra bambini che pesavano più o meno di $5 \mathrm{~kg}$, e tra bimbi con età gestazionale $<32$ settimane $\mathrm{e}$ tra 32 e 35 settimane.

In linea con quanto visto per l'endpoint primario, l'analisi dei dati relativi agli endpoint secondari ha evidenziato la riduzione nel gruppo palivizumab di parametri relativi alla severità dell'infezione da RSV, quali - rispettivamente - i giorni di ospedalizzazione per RSV, i giorni in cui si è dovuto somministrare ossigeno supplementare, e i giorni con malattia del tratto respiratorio inferiore da moderata a severa.

Va chiarito che i dati relativi alla terapia intensiva e alla ventilazione meccanica sono influenzati dalla bassa incidenza con cui si sono verificati questi eventi. A differenza di quanto rilevato con la formulazione endovenosa RSVIGIV nello studio PREVENT, l'incidenza di otite media non è risultata diminuita. L'incidenza di ospedalizzazione per motivi respiratori è risultata significativamente inferiore al gruppo placebo solo se venivano compresi anche gli eventi dovuti a RSV.

Le concentrazioni sieriche minime medie di palivizumab sono state di $37 \mu \mathrm{g} / \mathrm{ml}$ dopo l'iniezione iniziale e si sono mantenute $>40 \mu \mathrm{g} / \mathrm{ml}$ dopo quelle successive.

A livello di tollerabilità, eventi avversi giudicati correlati al farmaco sono stati rilevati nel $10 \%$ nel gruppo placebo e nell' $11 \%$ nel gruppo palivizumab. La differenza non è risultata significativa né per l'incidenza totale, né per quella specifica di ogni effetto collaterale ri- portato. Gli eventi avversi riportati in ordine di incidenza nel gruppo palivizumab sono stati: febbre, nervosismo, reazione locale nel sito di iniezione (generalmente lieve e di breve durata), diarrea, rash, aumento delle AST (aspartato aminotransferasi), malattia del tratto respiratorio superiore, anomalie nella funzione epatica (per lo più aumento contemporaneo di AST e ALT), aumento delle ALT (alanina aminotransferasi), vomito, tosse, rinite. L'uscita dal trial a causa di eventi avversi dovuti a palivizumab si verificata con estrema rarità $(0,3 \%)$ e i decessi verificatisi durante il trial non sono stati correlati a palivizumab (1,0\% nel gruppo placebo e $0,4 \%$ nel gruppo palivizumab).

A livello di immunogenicità, sono stati dosati i titoli di anticorpi anti-palivizumab, rilevandosi titoli $>1: 40$ nel 2,8\% del gruppo placebo e nell' $1,2 \%$ del gruppo palivizumab: si trattava comunque usualmente di riscontri isolati, presenti solo in una singola rilevazione, non collegati a specifici eventi avversi né a diminuzioni delle concentrazioni ematiche di palivizumab. D'altronde questa reattività, rilevata mediante ELISA (Enzyme-Linked ImmunoSorbent Assay) non pareva derivante da un' $\operatorname{IgG}$, e il riscontro di tale presenza anche nel gruppo placebo, non esposto cioè a palivizumab, suggerisce che l'evento non sia specifico. In ogni caso, in controlli effettuati a un anno dalla profilassi, non è più stato riscontrato questo anticorpo anti-palivizumab nel sangue dei bambini che avevano partecipato al trial.

Per fugare ulteriori dubbi è stato condotto uno studio open-label che ha valutato se le 5 somministrazioni di una seconda stagione della profilassi con palivizumab in 55 soggetti che l'avevano già ricevuto nello studio IMpact-RSV potessero causare problemi di immunogenicità o di scarsa tollerabilità [52]. Solo in un bambino su 55 è stato possibile rilevare un titolo anticorpale anti-palivizumab $>1: 40$, e comunque questo bambino non ha sviluppato eventi avversi gravi né si sono verificate riduzioni nella concentrazione minima sierica del farmaco. Lo studio dunque ha concluso che la profilassi con palivizumab per due stagioni epidemiche consecutive è sicura e ben tollerata.

Con riferimento ai bambini con grave patologia cardiaca di base, è stato progettato e condotto un grande studio multicentrico in doppio cieco denominato "Cardiac Synagis" per testare l'efficacia di palivizumab in tali popolazioni [47]. Detto studio ha arruolato 1.287 bambini con cardiopatia congenita emodinamicamente significativa con età $\leq 24$ mesi e li ha randomizzati 1:1 a ricevere placebo o 5 iniezioni IM mensili di palivizumab $15 \mathrm{mg} / \mathrm{kg}$. I soggetti, che sono stati seguiti per 150 giorni, potevano partecipare alla profilassi in una sola delle 4 stagioni del trial, durato dal 1998 al 2002.

I bambini con questa patologia erano già stati studiati nell'ambito della profilassi anti- 
RSV in due trial in cui era stata utilizzata una formulazione endovenosa (RSV-IGIV): quello condotto da Groothuis e colleghi nel 1993 [53] e quello di Simoes e colleghi del 1998 [54]. Nel primo caso, i pazienti affetti da cardiopatia congenita rappresentavano solo una parte del totale dei partecipanti al trial, e lo studio non era stato disegnato per effettuare un'analisi dei sottogruppi; tuttavia si poteva osservare una riduzione relativa di ospedalizzazioni da RSV del 63\% rispetto al gruppo placebo. Il secondo trial, che invece aveva reclutato solo bambini con cardiopatia congenita, aveva evidenziato un inatteso aumento di eventi avversi chirurgici in pazienti con patologia cianogena, forse causato da un'alterazione della viscosità ematica a seguito della somministrazione della profilassi con RSV-IGIV per via endovenosa in pazienti con ematocrito elevato [47].

A differenza di questi riscontri, il disegno dello studio Cardiac Synagis non è stato considerato rischioso per questa categoria di soggetti, in quanto la preparazione in uso di palivizumab ha un volume di 100 volte inferiore rispetto a RSV-IGIV e una quantità proteica di 50 volte più bassa.

Nello studio Cardiac Synagis, i bambini con cardiopatia congenita cianogena (682 cioè il 53\%) e non cianogena ( 605 cioè il $47 \%$ ) sono stati randomizzati in modo bilanciato tra soggetti riceventi palivizumab o placebo. L'endpoint primario, come già per lo studio IMpact-RSV, era l'ospedalizzazione da RSV: il risultato ottenuto è riportato nella Tabella VI, unitamente a quelli degli endpoint secondari.
La profilassi con palivizumab ha determinato una riduzione del rischio relativo di ospedalizzazione da RSV del 45\%. Tuttavia, benché si osservasse una riduzione del rischio di ospedalizzazione sia tra i soggetti cianogeni (riduzione del 29\%), sia tra quelli non cianogeni (riduzione del 58\%), tale riduzione risultava più marcata e statisticamente significativa nel secondo sottogruppo. La profilassi con palivizumab inoltre ha ridotto significativamente la durata (in giorni) dell'ospedalizzazione da RSV, e il numero di giorni di ospedalizzazione con necessità di ossigeno supplementare. Invece, per quanto riguarda gli altri parametri di severità di malattia, si è potuto solo osservare un trend verso la riduzione, che però non è stata significativa (Tabella VI).

La riduzione dell'11,8\% dell'incidenza di ospedalizzazione per qualsiasi causa dopo somministrazione di palivizumab, tuttavia, è risultata significativa $(p=0,008)$, ma, sottraendo i casi di ospedalizzazione per RSV, la significatività si perdeva $(p=0,056)$. I bambini con ospedalizzazione per motivi cardiorespiratori erano il $55,4 \%$ nel gruppo placebo e il 50,2\% nel gruppo palivizumab $(p=0,066)$. Le concentrazioni sieriche medie di palivizumab dopo la seconda e la quinta dose sono state rispettivamente di $55,5( \pm 19) \mu \mathrm{g} / \mathrm{ml}$ e di $90,8( \pm 35) \mu \mathrm{g} / \mathrm{ml}$, ma, nei soggetti che sono andati incontro all'intervento di bypass cardiaco, si è osservata una riduzione significativa del $58 \%$, che comportava una concentrazione di farmaco non più giudicata protettiva: per questo motivo gli Autori hanno concluso che dopo un intervento chirurgico è

\begin{tabular}{|c|c|c|c|c|}
\hline & $\begin{array}{l}\text { Palivizumab } \\
\text { (n = 639) }\end{array}$ & $\begin{array}{l}\text { Placebo } \\
(\mathrm{n}=648)\end{array}$ & $\begin{array}{l}\text { Riduzione } \\
\text { (\%) }\end{array}$ & $p$ value \\
\hline Incidenza di ospedalizzazioni da RSV & $34(5,3 \%)$ & $63(9,7 \%)$ & $45 \%$ & 0,003 \\
\hline Durata dell'ospedalizzazione per RSV & & & & 0,003 \\
\hline Giorni totali & 367 & 836 & & \\
\hline Giorni totali/100 bambini & 57,4 & 129,0 & 56 & \\
\hline $\begin{array}{l}\text { Durata di ospedalizzazione per RSV con } \\
\text { aumentato ossigeno supplementare }\end{array}$ & & & & 0,014 \\
\hline Giorni totali & 178 & 658 & & \\
\hline Giorni totali/100 bambini & 27,9 & 101,5 & 73 & \\
\hline Ospedalizzazioni in terapia intensiva & & & & 0,094 \\
\hline No & $626(98,0 \%)$ & $624(96,3 \%)$ & & \\
\hline Sì & $13(2,0 \%)$ & $24(3,7 \%)$ & 46 & \\
\hline Permanenza in terapia intensiva & & & & 0,080 \\
\hline Giorni totali & 101 & 461 & & \\
\hline Giorni totali/100 bambini & 15,9 & 71,2 & 78 & \\
\hline Necessità di ventilazione meccanica & & & & 0,282 \\
\hline No & $631(98,7 \%)$ & $634(97,8 \%)$ & & \\
\hline Sì & $8(1,3 \%)$ & $14(2,2 \%)$ & 41 & \\
\hline Ventilazione meccanica & & & & 0,224 \\
\hline Giorni totali & 42 & 354 & & \\
\hline Giorni totali/100 bambini & 6,5 & 54,7 & 88 & \\
\hline
\end{tabular}

Tabella VI

Riassunto dei principali risultati dello studio Cardiac Synagis (modificato da [47]) 


\begin{tabular}{lcc}
\hline & $\begin{array}{c}\text { Palivizumab } \\
\text { (\% incidenza) }\end{array}$ & $\begin{array}{c}\text { Placebo } \\
\text { (\% incidenza) }\end{array}$ \\
\hline Febbre & 27,1 & 23,9 \\
Infezione & 5,6 & 2,9 \\
Reazione nel sito di iniezione & 3,4 & 2,2 \\
Infezione delle vie aeree superiori & 47,4 & 46,1 \\
Congiuntivite & 11,3 & 9,3 \\
Aritmia & 3,1 & 1,7 \\
Cianosi & 9,1 & 6,9 \\
\hline
\end{tabular}

\section{Tabella VII}

Eventi avversi riportati con incidenza $\geq 1 \%$ nel gruppo palivizumab rispetto al gruppo placebo nello studio Cardiac Synagis [47]

opportuno somministrare un'altra dose di palivizumab.

Gli eventi avversi giudicati correlati al farmaco sono stati rari e quasi sempre del tutto comparabili nei due gruppi. Nessun bambino ha abbandonato il trial per eventi avversi. Pochi eventi avversi sono stati riportati come aventi un'incidenza $\geq 1 \%$ nel gruppo palivizumab rispetto al placebo, e sono riportati nella Tabella VII. Tuttavia, un solo evento di cianosi (ma rilevato nel gruppo placebo) $\mathrm{e}$ - fatto importante - nessun evento aritmico sono stati giudicati ascrivibili al trattamento. Va notato, per converso, che l'incidenza di eventi avversi gravi è stata significativamente inferiore nel gruppo palivizumab rispetto al placebo (354 eventi vs 409 , cioè $55,4 \%$ vs $63,1 \% ; \mathrm{p}=0,005)$; nel gruppo cianogeno e nel gruppo non cianogeno le riduzioni sono state confermate, ma con $\mathrm{p}$ value rispettivamente pari a 0,056 e 0,041 . Nessun evento avverso grave è stato imputato alla somministrazione di palivizumab, così come nessun decesso (mortalità complessiva:
$3,3 \%$ nel gruppo palivizumab e $4,2 \%$ nel gruppo placebo). I decessi attribuibili direttamente a RSV sono stati 2 nel gruppo palivizumab e 4 nel gruppo placebo.

\section{Sicurezza e tollerabilità}

Palivizumab non deve essere somministrato in caso di ipersensibilità accertata al principio attivo o a uno degli eccipienti della formulazione di Synagis ${ }^{\circledR}$ (istidina, glicina, mannitolo) o agli anticorpi monoclonali umanizzati [55].

Casi molto rari di anafilassi sono stati rilevati dopo somministrazione della profilassi con palivizumab, ragion per cui si consiglia di avere a disposizione farmaci per questa evenienza da poter usare subito dopo l'iniezione.

La profilassi mensile con palivizumab può essere rimandata in presenza di infezioni gravi o moderate o di affezioni febbrili; inoltre devono essere seguite le comuni norme di sicurezza per le iniezioni intramuscolari, usando cautela con i pazienti trombocitopenici o con i soggetti con altri disturbi della coagulazione. Non è certa, in quanto non indagata con studi ad hoc, l'efficacia del farmaco quando somministrato nella seconda stagione epidemica in bambini che avevano già ricevuto palivizumab in quella precedente.

Sebbene non siano stati condotti studi sulle interazioni con altri farmaci, dai trial effettuati è stato possibile notare un'uguale distribuzione di eventi avversi tra $i$ bambini che avevano ricevuto placebo e quelli a cui era stato somministrato palivizumab dopo vaccini di routine per l'infanzia, vaccino dell'influenza, broncodilatatori o corticosteroidi [55].

Per quanto riguarda gli eventi avversi, la distribuzione deve essere suddivisa a seconda delle categorie di pazienti studiati:

\section{Tabella VIII}

Effetti indesiderati verificatisi in studi clinici di profilassi su bambini prematuri o con displasia broncopolmonare in ordine decrescente di gravità (modificato da [55])

Comune $=$ frequenza maggiore o uguale di $1 / 100$ e minore di 1/10; non comune $=$ frequenza maggiore o uguale di $1 / 1.000$ e minore di $1 / 100$

\begin{tabular}{|c|c|c|}
\hline Tipo di problemi & Frequenza & Evento avverso \\
\hline \multirow[t]{2}{*}{ Infezioni e infestazioni } & Non comune & Infezione virale \\
\hline & & Infezione delle vie respiratorie superiori \\
\hline Disturbi del sistema ematico e linfatico & Non comune & Leucopenia \\
\hline Disturbi psichiatrici & Comune & Irritabilità \\
\hline \multirow[t]{3}{*}{ Disturbi respiratori, toracici e mediastinici } & Non comune & Sibili \\
\hline & & Rinite \\
\hline & & Tosse \\
\hline \multirow[t]{2}{*}{ Disturbi gastrointestinali } & Comune & Diarrea \\
\hline & Non comune & Vomito \\
\hline Disturbi della pelle e del tessuto sottocutaneo & Non comune & Eruzione cutanea \\
\hline \multirow[t]{3}{*}{ Disturbi generali e della sede di iniezione } & Comune & Febbre \\
\hline & & Reazione al sito di iniezione \\
\hline & Non comune & Dolore \\
\hline \multirow[t]{3}{*}{ Parametri di laboratorio } & Non comune & Aumento di AST \\
\hline & & Aumento di ALT \\
\hline & & Alterazione del test di funzionalità epatica \\
\hline
\end{tabular}




\begin{tabular}{lll}
\hline \multicolumn{1}{c}{ Tipo di problemi } & Frequenza & \multicolumn{1}{c}{ Evento avverso } \\
\hline Infezioni e infestazioni & Non comune & Gastroenterite \\
& Non comune & $\begin{array}{l}\text { Irritabilità } \\
\text { Disturbi psichiatrici }\end{array}$ \\
Disturbi del sistema nervoso & Non comune & Sonnolenza \\
& & Ipercinesia \\
Disturbi vascolari & Non comune & Emorragia \\
Disturbi respiratori, toracici e mediastinici & Non comune & Rinite \\
Disturbi gastrointestinali & Non comune & Vomito \\
& & Diarrea \\
& & Costipazione \\
Disturbi della pelle e del tessuto sottocutaneo & Non comune & Eruzione cutanea \\
& & Eczema \\
Disturbi generali e alla sede di & Comune & Febbre \\
somministrazione & & Reazione al sito di iniezione \\
& Non comune & Astenia \\
\hline
\end{tabular}

\section{Tabella IX}

Effetti indesiderati verificatisi in studi clinici pediatrici sulla profilassi nella malattia cardiaca congenita in ordine decrescente di gravità (modificato da [55])

Comune $=$ frequenza maggiore o uguale di $1 / 100$ e minore di $1 / 10 ;$ non comune $=$ frequenza maggiore o uguale di $1 / 1.000$ e minore di $1 / 100$
1. bambini prematuri o con broncodisplasia polmonare;

2. bambini con malattia cardiaca congenita.

In ogni caso, le reazioni descritte nei trial clinici sono state simili nei gruppi placebo e palivizumab, e la maggior parte di esse è stata transitoria e di gravità da lieve a moderata. L'interruzione della profilassi per causa di reazioni avverse è stata rara. Nelle Tabelle VIII e IX sono elencati gli eventi avversi giudicati causalmente correlati a palivizumab rispettivamente nella prima e nella seconda categoria di pazienti.

Oltre a quelli elencati nelle tabelle, sono stati riportati altri eventi avversi dopo l'immissione in commercio del farmaco (Tabella X): date le dimensioni incerte della popolazione di riferimento, risulta difficile stabilirne tanto la frequenza quanto la reale correlazione all'uso del farmaco. Gli eventi avversi registrati nel post-marketing a seguito di somministrazione di $>5$ dosi di palivizumab sono risultati comparabili per caratteristiche e frequenza a quelli già segnalati per un numero $\leq 5$ di somministrazioni.

Si è posta particolare attenzione all'immunogenicità di tale farmaco, per il timore che l'eventuale formazione di anticorpi leganti palivizumab potesse diminuire la concentrazione ematica del farmaco, formare immunocomplessi o generare immunotossicità diretta $[48,55]$ : in realtà anticorpi anti-palivizumab sono stati rilevati nell' $1 \%$ dei pazienti nella prima fase di terapia, ma si è comunque trattato di un fenomeno transitorio e a basso titolo.

\begin{tabular}{lc}
\hline \multicolumn{1}{c}{ Apparato coinvolto } & Evento avverso \\
\hline Sistema ematico e linfatico & Trombocitopenia \\
Sistema immunitario & Anafilassi \\
Sistema nervoso & Convulsioni \\
Distretti respiratorio, toracico e mediastinico & Apnea \\
Pelle e tessuto sottocutaneo & Orticaria \\
\hline
\end{tabular}

\section{Tabella $\mathbf{X}$}

Eventi avversi riportati a seguito della somministrazione di palivizumab dopo la sua immissione in commercio [55]

\section{CONCLUSIONI}

Dai dati clinici descritti si può concludere che palivizumab, oltre che sicuro, con eventi avversi comparabili al placebo, e maneggevole, per via della somministrazione intramuscolare, è in grado di ridurre il rischio di ospedalizzazione per RSV nelle categorie a rischio con un'efficacia che varia tra il $45 \%$ e il $55 \%$.

Bisogna considerare che il rischio di complicanze da RSV è alto, e che l'infezione severa può comportare delle ripercussioni respiratorie negli anni successivi. Se a questo si associano l'elevata mortalità in soggetti a rischio che costituiscono una discreta percentuale dei bambini sotto i due anni in Italia (si stima, ad esempio, circa il 5\% dei bambini prematuri e 1' $1 \%$ di quelli affetti da cardiopatia congenita), la dubbia o nulla efficacia delle terapie mediche disponibili per la bronchiolite da RSV, e l'assenza di un vaccino in commercio, appare chiara l'importanza di una profilassi efficace nel prevenire la malattia severa da RSV. 


\section{BIBLIOGRAFIA}

1. Eberle J. A treatise on the diseases and physical education of children. Philadelphia, PA: Lippincott, Grambo and Co.; 1850

2. Blount RE Jr, Morris JA, Savage RE. Recovery of cytopathogenic agent from chimpanzees with coryza. Proc Soc Exp Biol Med 1956; 92: 544-9

3. Groothuis JR, Hoopes M, Hemming VG. Prevention of serious respiratory syncytial virus-related illness: disease pathogenesis and early attempts at prevention. Adv Ther 2011; 28: 1-19

4. Heilman CA. Respiratory syncytial and parainfluenza virus. J Infect Dis 1990; 161: 402-6

5. Hall CB, Walsh EE, Schnabel KC, Long CE, McConnochie KM, Hildreth SW et al. Occurrence of groups A and B RSV over 15 years: associated epidemiologic and clinical characteristics in hospitalized and ambulatory children. J Infect Dis 1990; 162: 1283-90

6. Lanari M, Giovannini M, Giuffré L, Marini A, Rondini G, Rossi GA et al; the Investigators RADAR Study Group. Prevalence of Respiratory Syncytial Virus infection in italian infants hospitalized for acute lower respiratory tract infections, and association between Respiratory Syncytial Virus infection risk factors and disease severity. Pediatr Pulmonol 2002; 33: 458-65

7. American Academy of Pediatrics. Policy statement - modified recommendations for use of palivizumab for prevention of Respiratory Syncytial Virus infections. Pediatrics 2009; 124: 1694-701

8. Sung RYT, Murray HGS, Chan RCR, Davies DP, French GL. Seasonal patterns in respiratory syncytial virus infection in Hong. Kong: a preliminary report. J Infect Dis 1987; 156: 527-8

9. Tantivanich $\mathrm{S}$, Chityothin $\mathrm{O}$, Tharavanij $\mathrm{S}$. Infection rates of respiratory syncytial virus in pediatric patients attending Phra Mongkukklao Hospital, Bangkok. Southeast Asian J Trop Med Public Health 1984; 15: 63-7

10. Nwantko MU, Dym AM, Shnit KE, Offer E, Omene JA. Seasonal variation in respiratory syncytial virus infections in children in Benin-city, Nigeria. Trop Geogr Med 1988; 40: 309-13

11. Ruuskanen O, Ogra PL. Respiratory syncytial virus. Curr Probl Pediatr 1993; 23: 50-79

12. Dica33, glossario. Disponibile all'indirizzo http://www.dica33.it/servizi/glossario-medico.asp (ultimo accesso maggio 2011)

13. Kim HW, Arrobio JO, Brandt CD, Jeffries BC, Pyles G, Reid JL et al. Epidemiology of RSV in Washington, DC. I. Importance in different respiratory tract disease syndromes and temporal distribution. Am J Epidemiol 1973; 98 : 216-25

14. Hall CB, Powell KR, MacDonald NE, Gala CL, Menegus ME, Suffin SC et al. Respiratory syncytial virus infection in children with compromised immune functions. N Engl J Med 1986; 315: 77-81

15. De Boeck K. Respiratory syncytial virus bronchiolitis: clinical aspects and epidemiology. Monaldi Arch Chest Dis 1996; 51: 210-3

16. MacDonald NE, Hall CB, Suffin SC, Alexson C, Harris PJ, Manning JA. Respiratory syncytial viral infection in infants with congenital heart disease. N Engl J Med 1982; 307: 397-400

17. Groothuis JR, Gutierrez KM, Lauer BA. Respiratory syncytial virus infection in children with bronchopulmonary dysplasia. Pediatrics 1988; 82: 199-203

18. Hall CB, Kopelman AE, Douglas RG Jr, Geiman JM, Meagher MP. Neonatal respiratory syncytial virus infection. N Engl J Med 1979; 300: 393-6

19. Law BJ, de Carvalho V, PICNIC. Respiratory syncytial virus infections in hospitalized children: regional differences in patient populations and management practices. Pediatr Infect Dis J 1993; 12: 659-63

20. Wang EEL, Law BJ, Stephens D. Pediatric Investigators Collaborative Network on Infections in Canada (PICNIC) prospective study of risk factors and outcomes in patients hospitalized with respiratory syncytial virus lower respiratory tract infection. $J$ Pediatr 1995; 126: 212-9

21. Glezen WP, Paredes A, Allison JE, Taber LH, Frank AL. Risk of respiratory syncytial virus infections for infants from low income families in relations to age, sex, ethnic group, and maternal antibody level. J Pediatr 1981; 98: 708-15

22. Ruotsalainen M, Piippo-Savolainen E, Hyvarinen MK, Korppi M. Respiratory morbidity in adulthood after respiratory syncytial virus hospitalization in infancy. Pediatr Infect Dis J 2010; 29: 872-4

23. Stein RT, Sherrill D, Morgan WJ, Holberg CJ, Halonen M, Taussig LM et al. Respiratory syncytial virus in early life and risk of wheeze and allergy by age 13 years. Lancet 1999; 354: 541-5 
24. Sigurs N, Aljassim F, Kjellman B, Robinson PD, Sigurbergsson F, Bjarnason R et al. Asthma and allergy patterns over 18 years after severe RSV bronchiolitis in the first year of life. Thorax 2010; 65: 1045-52

25. Società Italiana di Neonatologia. Raccomandazioni della Società Italiana di Neonatologia per la prevenzione delle malattie da virus respiratorio sinciziale (VRS). Aggiornamento 2004. Acta Neonatologica \&Pediatrica 2004; 1 : 19-29

26. Giovannini M, Rossi GA, Merolla R, Arena F, Cutrera R, Dalla Casa P et al. Patologia respiratoria in bambini ospedalizzati nei primi due anni di vita per infezioni virali alle basse vie respiratorie: 1 anno di follow-up. Minerva Pediatr 2003; 55: 1-7

27. Medici MC, Arcangeletti MC, Merolla R, Chezzi C and the "Osservatorio VRS” Study Group. The "Osservatorio VRS" Study Group. Incidence of respiratory syncytial virus infection in infants and young children referred to the emergency departments for lower respiratory tract diseases in Italy. Acta Bio Medica Ateneo Parmense 2004; 75: 26-33

28. Di Carlo P, Romano A, Salsa L, Gueli A, Poma A, Fucà F et al. Epidemiological assessment of Respiratory Syncytial Virus infection in hospitalized infants, during the season 2005-2006 in Palermo, Italy. Ital J Pediatr 2009; 35 : 11

29. Simoes EA. Environmental and demographic risk factors for respiratory syncytial virus lower respiratory tract disease. J Pediatr 2003; 143: S118-S126

30. Van der Sande MA, Goetghebuer T, Sanneh M, Whittle HC, Weber MW. Seasonal variation in respiratory syncytial virus epidemics in the Gambia, West Africa. Pediatr Infect Dis J 2004; 23: 73-4

31. Liese JG, Grill E, Fischer B, Roeckl-Wiedmann I, Carr D, Belohradsky BH; Munich RSV Study Group. Incidence and risk factors of respiratory syncytial virus-related hospitalizations in premature infants in Germany. Eur $J$ Pediatr 2003; 162: 230-6

32. Openshaw PJ, Tregoning J, Harker J. RSV 2005: Global impact, changing concepts, and new challenges. Viral Immunol 2005; 18: 749-51

33. Kellner JD, Ohlsson A, Gadomski AM, Wang EE. Efficacy of bronchodilator therapy in bronchiolitis. A metaanalysis. Arch Pediatr Adolesc Med 1996; 150: 1166-72

34. Ventre K, Randolph A. Ribavirin for respiratory syncytial virus infection of the lower respiratory tract in infants and young children. Cochrane Database Syst Rev 2004: 181

35. Meert KL, Sarnaik AP, Gelmini MJ, Lieh-Lai MW. Aerosolized ribavirin in mechanically ventilated children with respiratory syncytial virus lower respiratory tract disease: a prospective, double-blind, randomized trial. Crit Care Med 1994; 22: 566-72

36. Moler FW, Steinhart CM, Ohmit SE, Stidham GL. Effectiveness of ribavirin in otherwise well infants with respiratory syncytial virus-associated respiratory failure. Pediatric Critical Study Group. J Pediatr 1996; 128: 422-8

37. Law B. Ribavirin does not reduce hospital stay (LOS) in patients with respiratory syncytial virus (RSV) lower respiratory tract infection (LRTI). Pediatr Res 1995; 37: 110A

38. Simoes EA. Immunoprophylaxis of respiratory syncytial virus: global experience. Respir Res 2002; 3 Suppl 1: S26-S33

39. Chanock RM, Parrott RH, Vargosko AJ, Kapikian AZ, Knight V, Johnson KM. Acute respiratory diseases of viral etiology. IV. Respiratory syncytial virus. Am J Public Health Nations Health 1962; 52: 918-25

40. Henderson FW, Collier AM, Clyde WA Jr, Denny FW. Respiratory-syncytial-virus infections, reinfections and immunity. A prospective, longitudinal study in young children. N Engl J Med 1979; 300: 530-4

41. Chin J, Magoffin RL, Shearer LA, Schieble JH, Lennette EH. Field evaluation of a respiratory syncytial virus vaccine and a trivalent parainfluenza virus vaccine in a pediatric population. Am J Epidemiol 1969; 89: 449-63

42. Fulginiti VA, Eller JJ, Sieber OF, Joyner JW, Minamitani M, Meiklejohn G. Respiratory virus immunization. I. A field trial of two inactivated respiratory virus vaccines; an aqueous trivalent parainfluenza virus vaccine and an alumprecipitated respiratory syncytial virus vaccine. Am J Epidemiol 1969; 89: 435-48

43. Kapikian AZ, Mitchell RH, Chanock RM, Shvedoff RA, Stewart CE. An epidemiologic study of altered clinical reactivity to respiratory syncytial (RS) virus infection in children previously vaccinated with an inactivated RS virus vaccine. Am J Epidemiol 1969; 89: 405-21

44. Kim HW, Canchola JG, Brandt CD, Pyles G, Chanock RM, Jensen K et al. Respiratory syncytial virus disease in infants despite prior administration of antigenic inactivated vaccine. Am J Epidemiol 1969; 89: 422-34

45. The PREVENT Study Group. Reduction of respiratory syncytial virus hospitalization among premature infants and infants with bronchopulmonary dysplasia using respiratory syncytial virus immune globulin prophylaxis. Pediatrics 1997; 99: 93-9 
46. The IMpact-RSV Study Group. Palivizumab, a Humanized Respiratory Syncytial Virus Monoclonal Antibody, reduces hospitalization from Respiratory Syncytial Virus infection in high-risk infants. Pediatrics 1998; 102; 531-7

47. Feltes TF, Cabalka AK, Meissner HC, Piazza FM, Carlin DA, Top FH, for the Cardiac Synagis Study Group. Palivizumab prophylaxis reduces hospitalization due to Respiratory Syncytial Virus in young children with hemodynamically significant congenital heart disease. J Pediatr 2003; 143: 532-40

48. EMEA. EPAR Synagis - Scientific Discussion. 2004. Disponibile all'indirizzo www.ema.europa.eu (ultimo accesso maggio 2011)

49. Informatore farmaceutico. Milano: Elsevier Masson, 2011

50. Subramanian KN, Weisman LE, Rhodes T, Ariagno R, Sánchez PJ, Steichen J et al. Safety, tolerance and pharmacokinetics of a humanized monoclonal antibody to respiratory syncytial virus in premature infants and infants with bronchopulmonary dysplasia. MEDI-493 Study Group. Pediatr Infect Dis J 1998; 17: 110-5

51. Sáez-Llorens X, Castaño E, Null D, Steichen J, Sánchez PJ, Ramilo O et al. Safety and pharmacokinetics of an intramuscular humanized monoclonal antibody to respiratory syncytial virus in premature infants and infants with bronchopulmonary dysplasia. The MEDI-493 Study Group. Pediatr Infect Dis J 1998; 17: 787-91

52. Null D Jr, Pollara B, Dennehy PH, Steichen J, Sánchez PJ, Givner LB et al. Safety and immunogenicity of palivizumab (Synagis) administered for two seasons. Pediatr Infect Dis J 2005; 24: 1021-3

53. Groothuis JR, Simoes EAF, Levin MJ, Hall CB, Long CE, Rodriguez WJ et al. Prophylactic administration of respiratory syncytial virus immune globulin to high-risk infants and young children. $N$ Engl J Med 1993; 329: 1524-30

54. Simoes EAF, Sondheimer HM, Top FH Jr, Meissner HC, Welliver RC, Kramer AA et al. Respiratory syncytial virus immune globulin for prophylaxis against respiratory syncytial virus disease in infants and children with congenital heart disease. J Pediatr 1998; 133: 492-9

55. RCP Synagis. Ultimo aggiornamento: 22/01/2010. Disponibile all'indirizzo http://www.ema.europa.eu/ema/index. jsp?curl=pages/medicines/human/medicines/000257/human_med_001070.jsp\&murl=menus/medicines/medicines. jsp\&mid=WC0b01ac058001d125 (ultimo accesso maggio 2011 ) 